\title{
La philosophie dans la presse écrite : le cas des entretiens
}

Philosophy in the written press: interviews as a case study

\section{Malika Temmar}

\section{(2) OpenEdition}

12 Journals

Édition électronique

URL : http://journals.openedition.org/aad/3115

DOI : 10.4000/aad.3115

ISSN : $1565-8961$

Éditeur

Université de Tel-Aviv

\section{Référence électronique}

Malika Temmar, «La philosophie dans la presse écrite : le cas des entretiens », Argumentation et Analyse du Discours [En ligne], 22 | 2019, mis en ligne le 15 avril 2019, consulté le 23 septembre 2019. URL : http://journals.openedition.org/aad/3115; DOI : 10.4000/aad.3115

Ce document a été généré automatiquement le 23 septembre 2019.

\section{cc) $(1) \ominus$}

Argumentation \& analyse du discours est mis à disposition selon les termes de la licence Creative Commons Attribution - Pas d'Utilisation Commerciale - Pas de Modification 4.0 International. 


\title{
La philosophie dans la presse écrite : le cas des entretiens
}

\author{
Philosophy in the written press: interviews as a case study
}

\author{
Malika Temmar
}

\section{Introduction}

1 En France, le discours philosophique existe d'abord comme discours académique, et il a la plupart du temps été étudié avec pour modèle ce seul type de discours; ce qui présuppose qu'un texte philosophique ne peut s'analyser qu'en adoptant le cadre restreint d'une discipline constituée. Nous voudrions élargir cette perspective pour tenir compte du fait que la philosophie s'est aussi très largement exportée hors des frontières strictes de l'enseignement et de la recherche. Une forte demande de philosophie de la part d'un large public lui confère une place de plus en plus grande dans la sphère médiatique. Or, on constate que rares ou inexistantes sont les études qui s'appuient sur les formes d'écriture adoptées par la philosophie quand elle sort de ces circuits de communication habituels et se voit adressée au «grand public» par la médiation de multiples supports: entretiens radiophoniques, télévisés, journaux, revues philosophiques grand public.

2 Cette contribution propose une analyse discursive des entretiens, interviews, «rencontres » avec un philosophe que publie la presse écrite. Ils s'inscrivent dans un cadre plus large, celui du recours journalistique aux paroles rapportées, aussi bien sous forme de citations que d'interviews ou encore de tribunes. L'une des particularités du discours journalistique consiste, en effet, à se faire le carrefour de plusieurs voix (Temmar 2014). Alors qu'à ses débuts le journal cherchait à faire entendre sa voix, celle-ci tend, à mesure que se professionnalise le journalisme, à se constituer autour la mise en scène de paroles extérieures (Moirand 2009).

3 Dans le texte de presse, la parole philosophique fait partie d'un ensemble comprenant aussi bien (à part quasi égale) celles du sociologue, de l'historien, de l'économiste, du psychologue et, de manière plus ponctuelle, de l'ethnologue, du politologue, de 
l'anthropologue. Quelques travaux portent sur la manière dont le discours des Sciences Humaines et Sociales se trouve transposé dans la presse. Outre l'étude de Habermas sur la publicisation des savoirs (1962), Eric Neveu (1995) a par exemple cherché à réfléchir sur les usages et les effets de l'«importation » des travaux de Sciences humaines et sociales dans l'espace public. On peut citer également la contribution de Boltanski et Thévenot (1991: 167-168). Mais on ne trouve pas l'équivalent pour la philosophie.

La parole philosophique a certes déjà donné lieu dans les médias à plusieurs recherches portant sur la figure de l'intellectuel engagé, ou sur celle du "penseur », mais non à une analyse de la manière dont cette parole est concrètement mobilisée. Que demandet-on au philosophe lors d'un entretien de presse? Est-ce qu'on s'entretient d'une manière particulière avec lui sur une question brûlante de l'actualité ? Quel portrait en construit-on à travers les questions qui lui sont posées lors de l'entretien? Ces questions le placent-ils en position de "conseiller» ou d'" expert", ou encore de prophète? Plus largement, nous nous demanderons dans quelle mesure l'entretien de presse peut nous éclairer en partie sur le rôle du philosophe dans le débat public, voire sur le rapport du philosophe à son époque.

Dans un premier temps, il nous faut considérer l'entretien avec un philosophe comme « genre », avant de nous consacrer à sa spécificité.

\section{La question de l'entretien}

6 Dans les médias, l'entretien répond à des critères d'analyse particuliers : comme le rappelle Jacques Mouriquand, « le projet de l'interview est de donner à entendre ce que tel ou tel peut avoir à dire sur un sujet » (2012: 70). Dans la presse écrite, qui nous intéresse ici, on assiste à un développement important de l'interview qui va jusqu'à marginaliser certains genres pourtant bien institués comme celui de la critique. Mouriquand explique ainsi ce phénomène :

On ne jugerait pas que ce soit sans rapport avec la pratique de la télévision. L'interview est facile à lire, d'abord parce qu'elle est le plus souvent courte, et que l'alternance de caractères différents pour marquer les questions et les réponses, dans le cas où l'on y recourt, facilite l'entrée du lecteur dans le texte. Ajoutons que c'est, en apparence, un exercice relativement simple à mener. Le risque principal étant celui de la banalité du propos [...]. Dans son édition du mercredi 3 avril 1996, le quotidien Le Parisien accorde de raisonnables développements à la sortie des films de ce mercredi-là. De façon tout à fait significative, chaque film est l'objet d'une interview (soit d'un acteur, soit d'un réalisateur), la critique faisant l'objet d'un court encadré toujours construit sur le même moule: une première partie consacrée à l'histoire, une seconde, à "notre avis ». Ce recours méthodique à l'interview, et la marginalisation corrélative d'un genre journalistique, la critique, longtemps tenue pour un des plus nobles, marque un virage significatif des évolutions récentes. Il fait penser à ces inévitables invitations d'acteurs sur les plateaux de télévision à l'occasion de la sortie de leur film (Mouriquand 1991 : 70).

\subsection{Les approches discursives de l'entretien}

7 L'entretien (ou l'interview, on trouve les deux appellations) comme " genre » discursif a donné lieu à de nombreuses études chez les interactionnistes. Elles le saisissent dans sa dimension essentiellement interactionnelle en privilégiant notamment les conflits, par exemple dans les débats politiques télévisés (Weizman 2013). Rares sont les 
approches des entretiens de presse chez les interactionnistes, qui préfèrent s'attacher aux entretiens oraux spontanés, non retravaillés par l'écrit.

8 Un numéro de Semen $(37,2014)$ a été consacré à l'étude du « récit biographique ». Il cherche à faire le lien entre l'analyse du discours et l'étude des entretiens mais n'aborde pas l'entretien de presse. Parce qu'il semble représenter un faux dialogue, qui donne une fausse impression de spontanéité, les travaux portant sur l'entretien de presse menés d'un point de vue discursif sont relativement peu nombreux. L'entretien de presse est par ailleurs souvent étudié quand on aborde la question des genres journalistiques (Adam 1997, Claudel 2010), et parfois par rapport au discours littéraire (Yanoshevsky 2014).

Dans ce qui va suivre, nous nous proposons d'étudier des entretiens suscités par la presse médiatique française (aussi bien la presse quotidienne nationale que la presse magazine) dans lesquels le philosophe est interviewé sur des questions d'actualité. Nous avons choisi de rassembler une série d'articles dans lesquels un philosophe est interrogé sur les attentats terroristes ${ }^{1}$.

\subsection{L'entretien portant sur des questions d'actualité}

Lorsque la presse s'entretient avec un philosophe, c'est souvent pour en appeler à la dimension de "bien vivre» que peut apporter la philosophie: selon la doxa, la philosophie serait «utile» pour mieux vivre. Il arrive aussi que le philosophe soit interrogé à propos de questions d'actualité. Dans ce cas, il est pour ainsi dire appelé sur la scène médiatique (au même titre que d'autres «experts ») pour répondre à des inquiétudes du moment.

11 Nous nous proposons d'étudier ici la manière dont le philosophe intervient et répond à cette demande sociale. Notre corpus est composé de quatorze entretiens avec des philosophes réputés. Nous choisissons cette thématique car elle présente l'intérêt de donner la parole au philosophe sur des « faits concrets » et parce que souvent, comme nous le verrons, on prête à la philosophie un « rôle » à jouer dans les grandes questions qui touchent la société. Ce thème pourrait faire écho à l'idéal des Lumières incarné dans cette phrase de Voltaire pour qui «il n'est d'autre remède à la maladie épidémique du fanatisme que l'esprit philosophique qui adoucit les mœurs des hommes et prévient les accès du mal » (1878 [1764] : 80). Le lien entre la question du terrorisme et la philosophie pourrait aussi être pensé par rapport non seulement à la thématisation du fanatisme, récurrente en philosophie, mais aussi par rapport à l'idée que celui-ci trouverait un remède grâce à la philosophie : comme le suggère Voltaire, chaque acte de barbarie, chaque génocide résulterait d'un déficit d'esprit philosophique.

\subsection{Les participants}

12 En apparence, deux instances sont en jeu dans l'entretien de presse avec un philosophe : l'intervieweur-journaliste et le philosophe interviewé, mais en réalité cette scène d'énonciation est composée de trois instances discursives puisque le lecteur, bien qu'absent, fait bel et bien partie du dispositif. C'est en effet au nom du lecteur que tout le dispositif de l'entretien fonctionne: c'est lui qui le formate puisqu'il s'agit de lui rendre accessibles des idées, une pensée. 

l'évidence un dialogue piégé. Le lecteur est toujours là. C'est en son nom que l'on va rencontrer un penseur, pour lui que chacun formule, l'un des questions, l'autre des réponses. C'est à son attention qu'est rédigé le texte final (Pol-Droit $2010: 49$ ). d'une fausse impression de spontanéité, puisque tout est retravaillé (contrairement aux entretiens sur lesquels travaillent les interactionnistes) : quand il y a un entretien en direct, il est vérifié par l'interviewé. C'est donc un texte semi-oral, voire totalement réécrit.

15 Les modalités d'écriture de l'interview varient selon les propos qui ont été tenus. Si l'éclairage retenu est très précis et que l'interlocuteur est éloquent, on aime souvent retracer l'entretien par une alternance de questions et de réponses. Les unes et les autres sont toujours réécrites pour ne garder que le plus intéressant. La réécriture des questions permet aussi de glisser des informations qui permettent de comprendre un contexte. Le journaliste est ici pris entre une absolue obligation de fidélité aux déclarations de son partenaire et l'absolue nécessité d'être parfaitement lisible pour son lecteur, ce second impératif ne devant jamais être oublié. Vieille règle journalistique : on n'écrit pas pour ses sources mais pour son public (Mouriquand 2013 : 64).

Dans cette dynamique de l'entretien, des places et des rôles se dessinent, une sorte d' ethos se construit aussi dans cette forme d'hétérogénéité énonciative (Yanoshevsky 2004). Le journaliste, à travers ses questions, construit une image du philosophe à qui il s'adresse. Il faut aussi s'interroger sur ce que recouvre l'image de l'intervieweur. Qu'est-ce que c'est qu'être un intervieweur de philosophe? Faut-il être un philosophe soi-même? De qui se fait-on l'écho? Parle-t-on en son nom propre ou au nom d'une pluralité et, si oui, laquelle ${ }^{2}$ ?

17 Comme le précise Mouriquand, dans l'entretien de presse l'objectif consiste pour l'intervieweur à extraire une information contenue dans le système des représentations de l'interviewé. On peut se demander quelle est la nature de cette information, s'agissant du philosophe. On serait a priori tenté de croire que s'entretenir avec un philosophe, c'est suivre une pensée qui se déroule à mesure qu'on l'écoute. C'est ainsi que Roger Pol-Droit définit d'ailleurs cette pratique, en en soulignant la singularité :

Plus intéressant me paraît de chercher ce qui se passe au juste au cours d'un entretien de ce genre. Mener un entretien avec un penseur, ce n'est pas la même démarche, me semble-t-il que s'entretenir avec un romancier, un comédien, un footballeur. Ce qui prime, n'est ni l'information, ni l'affectivité, il s'agit seulement d'inciter à réfléchir, à saisir des idées, de la manière la plus nette et simple, en pleine lumière [...]. Et pour quelques temps l'entretien s'installe. Le processus se déroule, dans son étrangeté toujours renouvelée. Car il y a quelque chose d'étranger dans cette pensée qui se déroule et se construit à mesure qu'on l'écoute. Rien à voir avec les dialogues où il s'agit d'énoncer les faits, ces entretiens en trompe l'œil qui ne sont que prospectus parlés ( «quand a eu lieu votre prochain concert? Où se tient votre exposition?») Rien à voir non plus avec ce que peut être un entretien avec un romancier (« pourquoi la mère d'Arthur l'emmène-t-elle à Marseille? », «le narrateur fait donc le même métier par hasard?", où il s'agit toujours plus ou moins d'entrer dans la fiction? $(2010: 45)$

18 Là, il est question d'idées. À faire partager, à rendre accessibles. Sans doute faut-il simplifier, forcer le trait, renoncer parfois à telle ou telle nuance. Mais il n'est jamais

Argumentation et Analyse du Discours, 22 | 2019 
question de trahir. Toute la difficulté tient à ceci : avancer à deux dans l'espace d'un dialogue, pour fabriquer des esquisses d'idées acceptables par un tiers dont on ne sait presque rien mais qui, en fait, bien qu'absent détermine le dispositif en totalité.

\subsection{Modalités de l'échange}

Tout entretien suit le modèle questions/ réponses. Ces interventions sont analysées par Blanchet de la manière suivante :

Le discours spécifique de l'intervieweur est constitué par un mode d'intervention que l'on appellera « relance » et dont on définira précisément les caractéristiques et le fonctionnement interlocutoire. Le discours spécifique de l'interviewé est constitué par ces deux caractéristiques de linéarité et de structuration. Le discours de l'interviewé est linéaire parce qu'il tend à être continu dans son enchaînement et à constituer un tout cohérent, apparemment non interrompu dans sa construction thématique par les interventions de l'intervieweur ; il est structuré propriété que Meyer - (1981) appelle plutôt "problématisé » et Laplanche (1987) « auto-théorisé » - dans la mesure où il tend à répondre à ses propres questions et ainsi à auto-entretenir sa propre production sous la stimulation des relances de l'intervieweur (1991:14).

Blanchet développe une théorie du principe actif de l'entretien de recherche que constitue la relance et de son mode d'action interlocutoire. Dans cette théorie, une notion est essentielle : celle d' « acte indirect» (1991: 14). De fait, dans notre corpus on repère de nombreuses réitérations qui sont des actes assertifs dont le contenu propositionnel est le même que le contenu propositionnel d'un énoncé de l'interviewé, selon les modèles de «l'écho », du "reflet ». Tout se passe comme s'il s'agissait parfois de préciser au lecteur non philosophe les propos du philosophe interviewé. Cette dimension didactique apparaît également dans les cas où le journaliste demande des exemples, des clarifications. Ces interventions sont à distinguer des déclarations qui obéissent au schéma suivant :

Complémentation : «vous avez dit $p$, je vous dis que j'en conclus $q$ »

Interprétation: «vous avez dit $\mathrm{p}$, je vous dis que j'en conclus que vous avez les propriétés-qualités X »

Dans ce cas, les interrogations sont des actes directifs dont le contenu propositionnel est inféré par l'intervieweur à partir de l'énoncé de l'interviewé selon les modalités suivantes :

Interrogations référentielles : « vous avez dit $\mathrm{p}$, je vous dis que j'en conclus q; est-ce que q est vrai?"

Interrogations modales: « vous avez dit $\mathrm{p}$, je vous dis que j'en conclus que vous avez les propriétés-qualités de $\mathrm{x}$; est-ce que $\mathrm{x}$ est vrai ?»

De manière générale, le type d'acte de relance de l'intervieweur est soit "réitératif", soit " déclaratif», soit « interrogatif». Ces relances traduisent une intention implicite de questionner, évaluer, voire de contester le propos de l'interviewé. Selon Labov et Fanshel (1977), l'intervieweur «challenge » ainsi l'interviewé dans son rôle et son statut de locuteur. Tout l'entretien vise au fond à faire admettre au fur et à mesure au journaliste et par extension au lecteur, une conclusion particulière. Ici, les stratégies d'orientation argumentatives ne manquent pas, marquées par de nombreux marqueurs lexicaux (topoï, connecteurs, adverbes évaluatifs, etc.).

Dans notre corpus nous avons constaté que la contre-argumentation, pourtant très à la mode dans l'entretien journalistique de ces dernières années, en particulier auprès 
d'hommes politiques, et qui s'ajuste assez bien aux situations dans lesquelles l'interviewé produit un plaidoyer public argumenté, est peu utilisée dans l'entretien avec le philosophe, comme s'il s'agissait moins de chercher à polémiquer qu'à apaiser les esprits. Dans ce cas, on est loin de la contre-argumentation où l'intervieweur est supposé relayer la parole du public ou d'un acteur qui ne peut pas énoncer ses propres opinions. Dans l'entretien avec un philosophe, la polémicité n'est mise en œuvre qu'avec certains philosophes très polémiques comme Michel Onfray, qui se veut provocateur dans ses prises de parole dans les médias.

\section{2. Étude du corpus}

Nous allons analyser dans un premier temps les modes de prise en charge énonciative à l'œuvre dans notre corpus. Pour répondre aux journalistes sur la question du terrorisme, le philosophe parle-t-il de manière impersonnelle (comme analyste) ou en son nom propre, ou encore au nom d'une autre instance, qui pourrait être par exemple «la » philosophie? Dans un deuxième temps, nous verrons les spécificités de ce type d'entretien, qui multiplie les modalités déontiques et l'appel aux valeurs. Cette particularité sera mise en regard de l'étude du type de questions posées systématiquement aux philosophes par les journalistes qui les érigent implicitement en "prophètes ». Nous verrons que ces valeurs déontiques posent la question de l'autorité au nom de laquelle parlent les philosophes. Ces entretiens présentent deux autres particularités : l'une consiste à avoir souvent recours aux définitions de mots, à une réflexion sur le lexique. Le philosophe, plus que n'importe quel autre expert, aura tendance à vérifier le rapport entre le mot et la chose. En second lieu, ces entretiens font intervenir de nombreux passages réflexifs sur le rôle de la philosophie quand il s'agit de penser l'événement.

\subsection{La prise en charge énonciative}

Au niveau énonciatif, dans l'ensemble du corpus les philosophes ont tendance à avoir recours au « je » embrayeur, accompagné très souvent d'un « nous » collectif (rarement d'un « on » omnipersonnel) opposé au groupe que forment «les terroristes ».-On peut s'en rendre compte dans ces passages ${ }^{3}$ :

Il me semble sensé d'adapter à la situation actuelle les deux dispositions de la Constitution française relatives à l'état d'urgence. Si cette question est désormais à l'ordre du jour, c'est parce que le président a proclamé l'état d'urgence à la suite des événements choquants de la nuit du 13 au 14 novembre, et entend le prolonger trois mois durant. Je peux difficilement juger de la nécessité de cette politique et de ses raisons. Je ne suis en rien un expert des questions de sécurité. Mais, envisagée à distance, cette décision ressemble à un acte symbolique permettant au gouvernement de réagir - vraisemblablement de la manière qui convient - au climat régnant dans le pays. En Allemagne, la rhétorique guerrière du président français, guidée semble-t-il par des considérations de politique intérieure, suscite des réserves.

Les experts sont d'accord sur ce point. Nous pouvons considérer ces barbares comme des ennemis, et nous devons lutter contre eux, inconditionnellement ; mais, si nous voulons vaincre cette barbarie sur le long terme, nous ne devons pas nous leurrer quant à ses raisons, qui sont complexes. 
J'ai bon espoir que la nation française donne au monde un exemple à suivre, comme elle l'a fait après l'attentat ayant visé Charlie Hebdo. (Habermas, Le Monde, 23 novembre 2015)

Tour à tour, Habermas se positionne comme «je » par rapport à d'autres instances discursives - ici les experts par exemple -, mais s'inscrit lui-même dans un "nous » collectif. On retrouve cela dans presque tous les textes étudiés.

- Pensez-vous que cette tragédie va créer un malaise avec la communauté musulmane en France?

- Je ne crois pas que la petite politique proposée par Hollande soit de nature à peser sur l'Histoire. Il nous faudrait une grande politique dont il n'est pas capable - dont il n'a jamais été capable et dont il ne sera pas capable. (Onfray, « La France doit cesser sa politique islamophobe », Le Point, 15 novembre 2015)

Debray ajoute à ce « nous » collectif un « on » :

En dehors de l'intérêt du consommateur, et du taux de croissance, nous ne voyons qu'irréalités fumeuses et subjectives. On ne comprend donc rien à ce qui nous arrive. Le libéralisme économique à tous crins est viable aux États-Unis parce qu'il y a là-bas une épine dorsale, une religion civile, biblico-patriotique. (Le Figaro.fr, 3 oct. 2016)

Si le « je » embrayé est très présent dans la plupart des cas (à l'exception de Debray, qui dans le corpus étudié a recours plus systématiquement au « on »), il réfère au locuteur dans sa singularité. Dans les textes étudiés, celui d'Onfray est très marqué, comme celui de Bernard-Henri Lévy, qui va jusqu'à se permettre l'expression d'une émotion : «je suis atterré ».

- Le phénomène kamikaze vient encore de frapper. Mais, cette fois, en Israël. Quelle est votre réaction?

- Je suis atterré. D'abord parce que, encore une fois, j'ai étudié de près ce phénomène kamikaze et que c'est l'arme face à laquelle les démocrates sont et seront toujours le plus démunis (Le Figaro, 8 décembre 2001).

Dans le même sens on trouve chez Onfray :

- La France doit-elle se désolidariser de la coalition internationale engagée en Syrie et en Irak?

- Je suis en effet partisan d'une remise à plat totale de la politique étrangère française. Si nous continuons à mener cette politique agressive à l'endroit des pays musulmans, ils continueront à riposter comme ils le font. Envoyer des troupes au sol en Syrie serait répandre des fleuves d'huile sur le feu. La France devrait cesser cette politique néocoloniale et islamophobe alignée sur les États-Unis (Le Point.fr, 15 novembre 2015).

traste, Roger-Pol Droit questionne ce « je » en posant clairement la question de savoir s’il désigne le philosophe interrogé comme représentant de « la » philosophie, ou comme « simple subjectivité » :

En outre, je ne saurais parler au nom de « la » philosophie, qui est multiple, diverse, traversée de quantités d'écoles et de querelles. Je ne revendique donc que mon regard, celui d'une subjectivité qui s'efforce de réfléchir, de discerner des éléments à creuser dans le bruit et la fureur de l'actualité. (La Tribune, 1 février 2015)

1 Dans les différents textes étudiés, les philosophes les plus médiatisés ont tendance à marquer plus explicitement leur subjectivité en affichant des prises de position fortes et en mettant en scène une émotion. C'est le cas, on l'a vu, de Bernard-Henri Lévy. Onfray, de son côté, fait part de ses propres inquiétudes :

Hélas, oui, je le crains. Je le crains d'autant plus que nombre d'individus ont intérêt à faire s'effondrer le système démocratique en France, de l'extrême gauche à l'extrême droite, la vraie, et que, monter les Français contre l'ensemble de la 
communauté musulmane s'avère facile et de rapport direct, malheureusement (Le Point.fr, 15 novembre 2015).

Bien que les textes soient pris en charge selon des modalités différentes, dans les réponses des philosophes - y compris ceux dans lesquels la subjectivité est moins visible -, ce sont les modalités déontiques qui dominent.

\subsection{Les modalités déontiques}

Ces modalités expriment ici pour la plupart l'obligation, le devoir; le conseil et la recommandation sont également fréquentes. La modalité aléthique y est représentée par les jugements de nécessité exprimés le plus souvent par le verbe impersonnel " falloir » et les expressions impersonnelles comme « il convient de », « il est nécessaire de ». On peut s'en rendre compte avec ces exemples:

- La société civile doit se garder de sacrifier sur l'autel de la sécurité toutes ces vertus démocratiques d'une société ouverte que sont la liberté de l'individu, la tolérance vis-à-vis de la diversité des formes de vie et la bonne disposition à adopter la perspective d'autrui. (Habermas, Le Monde, 23 novembre 2015)

- Il faut arrêter de donner une noblesse politique à ces actes de psychose collective, provoqués par des consciences totalement aliénées, sous-éduquées, qui ne connaissent rien à l'herméneutique religieuse, qui sont souvent sous drogues ou substances chimiques, et qui utilisent des armes de barbarie tout à fait classiques, basiques, comme le viol et la torture. Je ne crois pas qu'il soit pertinent de légitimer ces actions en leur donnant une quelconque valeur idéologique ou intellectuelle. (c. Fleury, Le Point, 26 novembre 2015)

- Mais les citoyens doivent être aussi dans leur rôle en retenant, par leur vigilance et leur puissance critique, l'exécutif dans sa pente sécuritaire - par exemple en refusant de considérer que l'exception puisse devenir permanente. Face au terrorisme, la participation de tous à l'effort de sécurité est requise, mais il y a sans doute autre chose à cultiver en soi que la défiance et la suspicion. La résistance éthique, le refus de se constituer soi-même comme sujet sécuritaire, est fondamentale aussi, sont l'honneur du sujet politique en démocratie. (Gros, Le Monde, 21 novembre 2015).

- Les politiques doivent marteler que Daech n'est pas l'islam. Après la condamnation absolue du crime et la compassion pour les victimes, nous devons entrer dans le temps de la réflexion et de l'analyse. Ces attentats sont un «épiphénomène européen » - certes insupportable - d'une guerre terrible qui se joue à l'intérieur de l'islam : dans le monde, les premières victimes en sont les musulmans eux-mêmes. (Crépon, L'Obs., 17 décembre 2015)

- Le terrorisme comme la crise des réfugiés constituent des défis dramatiques, peut-être ultimes, et exigent une coopération étroitement solidaire à laquelle les nations européennes ne se sont jusqu'à présent pas encore résolues, y compris dans le cadre de l'union monétaire.

La France devrait cesser cette politique néocoloniale et islamophobe alignée sur les ÉtatsUnis. Elle devrait retirer ses troupes d'occupation dans tous les endroits concernés. Elle devrait prendre l'initiative d'une conférence internationale qui viserait à constituer un front diplomatique à même de négocier une neutralité associée à un respect de la souveraineté politique de chacun de ces pays qui ont le droit de faire ce qu'ils souhaitent sur leur territoire sans que nous le leur interdisions. (M. Onfray, Le Point.fr, 15 novembre 2015)

- Comment doit, selon vous, réagir la communauté internationale?

- La première chose à faire, c'est de mettre Arafat au pied du mur. On ne peut pas à la fois condamner le terrorisme en paroles et l'encourager en sous-main. On ne peut pas faire sortir de prison, comme il l'a fait l'an dernier, les plus sanguinaires des assassins du Hamas et feindre de s'étonner, maintenant, de les voir passer à l'acte. Pire : on ne 
peut pas prétendre à la direction d'un peuple si on passe son temps à dire qu'on ne sait rien, qu'on ne peut rien, qu'on ne contrôle rien. Ça, c'est la première chose. C'est l'urgence absolue. (B-H. Lévy, Le Figaro, 8 décembre 2001).

On est frappé à la lecture de ces extraits par la manière dont les philosophes énoncent des injonctions, posent une action à faire ou ne pas faire, en se donnant une autorité particulière. Ces injonctions sont en fait suscitées et consolidées par les questions posées au philosophe par le journaliste.

\subsection{L'image du philosophe construit par le journaliste}

L'analyse du type de questions posées par les journalistes au philosophe est intéressante à considérer pour comprendre l'image du philosophe qui se construit à travers l'interview.

Ces questions peuvent être classées selon différents types. Tour à tour le philosophe est interrogé sur une «manière de faire » ouverte par des questions qui commencent par «comment?", sur sa capacité analytique, ce qu'«il pense de »... Une autre série de questions l'interrogent sur l'état du monde.

- À F. Gros :

Mais comment peut-on résister à ce que vous nommez des « guerres diffuses »?

Comment résister, intérieurement et éthiquement, à cette terreur? (Le Monde, 21

novembre 2015)

- À Bernard-Henri Lévy :

Comment expliquez-vous que les talibans aient connu une déroute aussi rapide?

Comment envisagez-vous les suites de la guerre?

Comment doit, selon vous, réagir la communauté internationale? (Le Figaro, 8 décembre 2001)

Ces questions contrastent avec une série d'autres introduites par «que pensez vous de », qui semblent quant à elles solliciter le philosophe dans sa seule capacité d'analyse, dans sa posture de penseur.

- Le président François Hollande veut définir un «état de guerre » adapté à la situation. Que pensez-vous de cette discussion? Croyez-vous plus généralement qu'une modification de la Constitution soit une réponse adaptée aux attentats du 13 novembre?

- Le président Hollande a aussi décidé d'accroître son niveau d'intervention en Syrie, notamment en bombardant Rakka, la « capitale » de l'Etat islamique, et en se rapprochant de la Russie. Que pensez-vous de l'interventionnisme en général? (A. Habermas, Le Monde, 21 novembre 2011)

Ces questions contrastent aussi avec celles, plus nombreuses, qui placent le philosophe dans une situation particulière par rapport à d'autres instances discursives: le journaliste (et à travers lui l'instance discursive dont il se fait le représentant) prête au philosophe le pouvoir d'accéder à une sorte de vérité ultime.

- «Le monde contemporain se trompe?» (A R. Debray, Le Figaro.fr, 3 octobre 2015)

- «La laïcité peut-elle répondre au désir de sacré de l'individu? » (A R. Debray, Le

Figaro.fr, 3 octobre 2016)

- «Leur radicalisation obéit-elle à un choix rationnel ?» (A M. Onfray, Le Point.fr, 15

novembre 2015)

- «Quelle est la source de nos maux ?» (A R. Debray, Le Figaro.fr, 3 octobre 2016)

Ce pouvoir d'éclairer culmine dans certains passages en un pouvoir quasi prophétique, où l'on demande au philosophe de « prédire » le futur. 
- Mesure-t-on la nature, l'envergure, le sens de l'empreinte que cet événement laissera dans l'histoire de France, et aussi dans celle de la construction identitaire et religieuse de la «nation française »?

L'islamophobie n'est plus la seule propriété des formations politiques d'extrême droite. Comme cela est démontré chaque lundi en Allemagne sous la coupe du mouvement Pegida, les citoyens s'en sont emparés. Ce changement de paradigme augure-t-il une cristallisation croissante au sein de la population, et le déplacement des fractures ethniques, religieuses, sociales sur un terrain alors politiquement incontrôlable? (A R-P. Droit, La Tribune, $1^{\text {er }}$ février 2015)

- Face à de tels enjeux, que faut-il espérer ou exiger des deux terrains principaux d'apprentissage ou d'exercice du vivre-ensemble : l'école et l'entreprise? (A R-P. Droit, La Tribune, $1^{\text {er }}$ février 2015)

- Assiste-t-on, avec ces attentats, à un traumatisme collectif inédit?

Se rassembler, se prendre en photo sur les terrasses, brandir " Paris est une fête ", c'est une réponse dionysiaque pertinente? (A C. Fleury, Le Point, 26 novembre 2015)

C'est le journaliste qui érige le philosophe en prescripteur. Il en fait tour à tour un conseiller, et un prophète. Si on lui demande un diagnostic sur le monde, souvent le philosophe est perçu comme doté d'un pouvoir qui serait d'être à la fois dans la société et en dehors d'elle. Aussi attend-on du philosophe des " remèdes ", des solutions.

Dans la plupart des cas, il est difficile de situer l'autorité au nom de laquelle il énonce. Sa parole apparaît comme autorisée sans qu'il soit possible de retrouver l'origine de sa légitimité énonciative. Les termes par lesquels cette autorité se désigne sont des rappels récurrents aux valeurs.

- La société civile doit se garder de sacrifier sur l'autel de la sécurité toutes ces vertus démocratiques d'une société ouverte que sont la liberté de l'individu, la tolérance vis-à-vis de la diversité des formes de vie et la bonne disposition à adopter la perspective d'autrui. En face d'un Front national qui se renforce, cela est plus facile à dire qu'à faire. (J. Habermas, Le Monde, 21 novembre 2015)

- Aucune parole de vérité n'est destructrice, juge le philosophe, qui exhorte à "dire, dire, dire" pour éteindre les feux, libérer les consciences, restaurer les vertus de la confrontation même conflictuelle à l'autre et à soi, se laisser interpeller par les mystères de la complexité, et ainsi revitaliser un vivre-ensemble disloqué mais que la mobilisation citoyenne à l'issue des attentats de Charlie Hebdo et de l'Hyper Casher a montré la volonté de réparer. Cette acceptation de la « complexité » constitue chez Roger-Pol Droit le fil rouge de ses explorations intellectuelles et de ses recommandations, y compris pour justifier les trésors que recèle l'entreprise et pour circonscrire «sa » définition et «sa" vocation de la philosophie, fondamentalement hostiles au dogme du «bonheur» qui l'a travestie. «Chercher à construire une doctrine du bonheur est une négation de la vie, et espérer une vie sans négatif est un mirage.» Toutes les formes de fanatismes prospérant dans le rejet des dualités, des ambivalences, des antagonismes "intérieurs", ensemencer un enseignement durable, solidaire, humaniste des événements de janvier 2015 exige l'acceptation universelle de cette complexité. Société et entreprises y sont-elles préparées? (D. Lafay, « À propos de Roger-Pol Droit ", La Tribune, $1^{\text {er }}$ février 2015)

\subsection{Le recours à la réflexion sur le lexique comme geste spécifiquement philosophique}

Dans un long entretien, Derrida, interrogé par un journaliste sur la différence entre un intellectuel et un philosophe avance que, pour lui, le philosophe est celui qui prendra toujours de la distance par rapport au langage ; d'où son souci de distinguer différentes significations des termes, de démêler les sens multiples et parfois embrouillés de 
concepts porteurs d'une longue histoire, de faire ressortir leurs diverses faces, montrer en quoi elles se différencient et parfois se superposent.

C'est une question difficile parce que vous le savez très bien, le concept d'intellectuel a une énorme histoire, pour une large part liée d'ailleurs, à l'histoire française. On a rarement, dans la définition française de l'intellectuel, dissocié l'intellectuel du philosophe, de Voltaire à Sartre ou même à Zola... Il était intellectuel dans la mesure où il n'était pas simplement un artiste, un écrivain, un esthète, mais dans la mesure où il posait des questions de responsabilité métaphysique, éthique, politique... qui ont une dimension philosophique.

D'une certaine manière, il est difficile de distinguer radicalement l'intellectuel du philosophe. Cela dit, dans la grande famille des intellectuels, certains font profession de philosophie. Ils ont donc en principe, un goût et un entrainement particuliers qui font que, d'une part, ils ont la mémoire de l'histoire de la philosophie, du style de la question philosophique, de ce qu'elle doit être et, d'autre part, ils prétendent justement, ce que ne font pas tous les intellectuels, à la plus grande vigilance quant à l'histoire des concepts qu'ils utilisent - l'invention des concepts politiques, par exemple -, a une responsabilité quant à la provenance et la pertinence des concepts qu'ils utilisent. Cela, tous les intellectuels ne l'ont pas, quelques soient leurs qualités. D'autre part, le philosophe en tant que philosophe a à prendre de la distance, que je crois légitime par rapport au langage dans lequel la discussion intellectuelle est établie. (Derrida, Le Point.fr, 9 novembre 2014)

Dans les entretiens étudiés, la plupart des textes montrent en effet un effort pour sans cesse interroger le philosophe sur l'adéquation entre le mot et la chose. De nombreux passages en témoignent :

- Pensez-vous que nos dirigeants aient eu raison de parler de "guerre » à la suite des attentats terroristes du 13 novembre?

- Nous sommes aujourd'hui loin des guerres dites «conventionnelles». Mais comment caractérisez les nouveaux conflits? (A F. Gros, Le Monde, 21 novembre 2015).

- Devant les attaques terroristes que la France a subies, reprenez-vous à votre compte le lexique de la guerre?

Vous avez introduit la notion de «fait religieux » en France. Est-elle bien abordée? (A R. Debray, Le Figaro, 3 octobre 2016).

- «Sommes-nous vraiment en guerre? » (A M. Onfray, Le Point.fr, 15 novembre 2015)

- Giovanna Borradori : Vous croyez qu'on peut marquer ces distinctions? Les définitions courantes n'excluent donc pas le «terrorisme d'Etat». Tous les terroristes prétendent y répliquer [...].

G. B. Qu'entendez-vous au juste par terrorisme? Peut-on sensément distinguer un terrorisme national d'un terrorisme global ? [...]

G. B. Doit-on distinguer le terrorisme des crimes habituels et des autres formes de recours à la violence? [...]

G.B. Croyez-vous que ce fut une bonne chose d'interpréter cet acte comme une "déclaration de guerre?"

- Jacques Derrida - Sans doute un tel "événement» requiert-il une réponse philosophique. Mieux, une réponse qui remette en question, dans leur plus grande radicalité, les présuppositions conceptuelles les mieux ancrées dans le discours philosophique. Les concepts dans lesquels on a le plus souvent décrit, nommé, catégorisé cet «événement " relèvent d'un «sommeil dogmatique » dont ne peut nous réveiller qu'une nouvelle réflexion philosophique, une réflexion sur la philosophie, notamment sur la philosophie politique et sur son héritage. Le discours courant, celui des médias et de la rhétorique officielle, se fie trop facilement à des concepts comme celui de "guerre » ou de «terrorisme " (national ou international).

Une lecture critique de Carl Schmitt (1), par exemple, serait fort utile. D'une part, pour prendre en compte, aussi loin qu'il est possible, la différence entre la guerre 
classique (confrontation directe et déclarée entre deux Etats ennemis, dans la grande tradition du droit européen), la "guerre civile» et la «guerre des partisans» (dans ses formes modernes, encore qu'elle apparaisse, Schmitt le reconnaît, dès le début du XIX ${ }^{\mathrm{e}}$ siècle).

Mais, d'autre part, il nous faut aussi reconnaître, contre Schmitt, que la violence qui se déchaîne maintenant ne relève pas de la guerre (l'expression " guerre contre le terrorisme " est des plus confuses, et il faut analyser la confusion et les intérêts que cet abus rhétorique prétend servir). (Le Monde diplomatique, février 2004, p. 18) sur le lexique ou dans lesquels le philosophe insiste sur le fait de ne pas utiliser de manière abusive tel terme. Il s'agit de mimer ici tout le travail philosophique qui consiste à montrer une extrême "vigilance" quant aux termes employés. Cette attention est d'autant plus importante quand, autour des attaques terroristes, certains ont avancé le mot de « guerre » pour qualifier ces actes. Ainsi R.-P. Droit :

- À l'évocation des attentats en France mais aussi des décapitations perpétrées par Daech ou des enlèvements organisés par Boko Haram, une dénomination est communément retenue pour qualifier les auteurs et leurs actes: barbares et barbarie. En 2007, vous avez publié un ouvrage, Généalogie des barbares (Odile Jacob), qui explore l'évolution, depuis les Grecs, des représentations de la barbarie. Cette projection dans le temps lointain et son étude historique nous apprennent-elles à « lire » la réalité de la barbarie en 2015 ?

- Au moins d'une lumière frisante, car ces notions de barbares et de barbarie ont effectivement une longue histoire qui permet de comprendre différemment les usages actuels. Les Grecs ont inventé le terme, et l'idée même de "barbare». Mais la notion de "barbarie », au sens de l'inhumanité dans l'homme, leur était totalement étrangère ! Pour les Grecs de l'Antiquité, les barbares sont d'abord des gens qui « parlent mal ». C'est là le sens originaire du terme, qui est attesté chez Homère. Mais on ne peut "parler mal " sans être aussitôt soupçonné de "mal penser», du moins dans le contexte grec, où l'étroite parenté de la parole et de la raison s'inscrit dans le terme même de logos, qui signifie les deux, parole et raison. (R.-P. Droit, La Tribune RhôneAlpes, $1^{\text {er }}$ février 2015)

Dans la mesure où il montre une rigueur particulière dans son analyse des termes, le philosophe serait plus apte à accéder à l'identification de ce qui surgit dans l'actualité.

\subsection{Réflexivité : penser l'événement}

On trouve dans les entretiens une constante réflexivité : l'entretien consolide la place majeure de la philosophie dans le débat public et la vie de tous les jours. Cela est particulièrement perceptible dans les entretiens où la philosophie est prise pour thème : ce qui est alors interrogé c'est son pouvoir, son utilité. La philosophie est présentée comme une discipline qui serait indispensable.

Le travail du journaliste est de commenter ce qui advient, celui du philosophe, de mettre en perspective ce qui est avec les conditions qui ont rendu possible ce qui advient. Le chef de l'État parle d'« acte de guerre ». Les Républicains et le Parti socialiste aussi. Tout le monde semble enfin convenir qu'il s'agit d'actes de guerre. C'est déjà un progrès ! Il y a peu, on parlait encore d'actes commis par des déséquilibrés, de gens au passé psychiatrique lourd, de loups solitaires. Dès lors qu'il s'agit de guerre, il faut la penser. Le journalisme télé a moins le souci de penser la guerre que de mettre en scène le spectacle de la terreur et de le commenter en se contentant de dire ce que chacun voit à l'écran. Le philosophe se demande d'où elle vient. Qui l'a déclarée? Quand? Pourquoi? Quels sont les belligérants? Quelles sont leurs raisons? Il faut dès lors sortir du

Argumentation et Analyse du Discours, 22 | 2019 
temps court du journaliste qui vit d'émotion pour entrer dans le temps long du philosophe

qui vit de réflexion. (M. Onfray, Le Point.fr, 15 novembre 2015) veut prescripteur. Discours normatif, il se rapproche sur ce point du discours religieux. Une analyse de la prise de parole du Pape sur la question du terrorisme montre des similitudes: il tient un discours qui se veut d'apaisement, qui recherche l'harmonie plutôt que la polémique.

Nous n'avons pas cherché à distinguer ceux qui, parmi les interviewés, sont des philosophes académiques et ceux dont l'existence est essentiellement médiatique. Malgré leurs profils différents, il existe des invariants dans la manière d'aborder la question du terrorisme.

Faire intervenir un philosophe sur des événements qui saturent les médias à un moment donné pose des questions intéressantes sur la temporalité philosophique : le philosophe est celui qui répond avec du recul. Quand il intervient dans la presse, il risque, comme le rappelle Derrida, d'être confronté à un problème de double temporalité. Les interventions courtes, immédiates, parce qu'elles se glissent dans un code relativement commun ont une chance d'être assez vite entendues. Les autres, celles que Derrida appelle "radicales", peuvent rencontrer des destinataires en nombre limité, relativement peu visibles, formés d'une certaine manière et qui seront les médiateurs, eux aussi incertains, d'une parole qui ne résonne pas immédiatement. Ce n'est donc pas seulement une question de rythme, c'est aussi une question de destinataires et d'audience.

\section{BIBLIOGRAPHIE}

Adam, Jean-Michel. 1997. « Genres de la presse écrite », Pratiques 94, 4-9

Blanchet, Alain. 1991. Dire et faire dire l'entretien (Paris : Colin)

Boltanski, Luc \& Laurent Thevenot. 1991. De la justification : les économistes de la grandeur (Paris : Gallimard)

Claudel, Chantal. 2010. « Les pratiques journalistiques en France et au Japon : points de rencontre et divergences ", Communication 164, 13-20

Droit, Roger-Pol. 2010. La compagnie des philosophes (Paris : Odile Jacob)

Ducrot, Oswald \& Jean-Claude Anscombre. 1983. L'argumentation dans la langue (Liège : Mardaga)

Argumentation et Analyse du Discours, 22 | 2019 
Heinich, Nathalie. 2002. « Pour une neutralité engagée », Questions de communication 2, 117-127

Labov, William \& David Fanshel. 1977. Therapeutic discourse (New York: Academic Press)

Mehl, Dominique. 2003. La Bonne parole. Quand les psys plaident dans les médias (Paris : La Martinière)

Moirand, Sophie. 2007. Les discours de la presse quotidienne. Observer, analyser, comprendre (Paris : PUF)

Mouriquand, Jacques. 2013. L'Écriture journalistique (Paris : PUF)

Weber, Max. 1959. Le savant et le politique (Paris : Plon)

Neveu, Eric. 1995. « Les sciences sociales face à l'espace public, les sciences sociales dans l'espace public », Paillart, Isabelle (éd.), L'espace public et l'emprise de la communication (Grenoble : Ellug), 37-64

Temmar, Malika. 2014. « Die Debatte um das französische Hochschulgesetz L.R.U. von 2009. Eine Äußerungsanalyse der Darstellung hochschulpolitischer AkteurInnen in Pressetexten », Johannes Angermüller et al. (eds.), Diskursforschung. Ein interdisziplinäres Handbuch (Bielefeld : Transcript), 454-471

Voltaire. 1878 [1764]. Dictionnaire philosophique, article "Fanatisme" (Paris : Garnier)

Weisman, Elda. 2013. The pragmatics of political discourse: explorations across cultures (Augsburg: Benjamins)

Yanoshevsky, Galia. 2004. «L'entretien d'écrivain et la co-construction d'une image de soi : le cas de Nathalie Sarraute », Revue des sciences humaines 273 (1), 131-148

Yanoshevsky, Galia. 2014. «L'entretien littéraire - un objet privilégié pour l'analyse du discours ? », Argumentation et Analyse du Discours 3; http://journals.openedition.org/aad/1726

\section{Corpus}

Badinter, Elisabeth. «Quelle République face à l'islamisme ?» 2001. Le Monde.fr, 2 avril. En ligne: https://www.lemonde.fr/idees/article/2016/04/02/quelle-republique-face-a-l-

islamisme_4894548_3232.html (consulté le 18 octobre 2017)

Lévy, Bernard-Henri (entretien avec Catherine Nay et Patrice de Meritens). 2001. « De New York à Jérusalem, le terrorisme le plus aveugle semble laisser le monde démuni », Le Figaro, 8 décembre 2001, p. 43

Bruckner, Pascal (entretien avec Jean-Christophe Marmara). 2016. «Face aux djihadistes, la naïveté n'est plus permise », Le Figaro, 21 déc. 2016, p. 14

Crépon, Marc (entretien avec Cécile Deffontaines). 2015. «L'état d'urgence est contre-productif à long terme ", L'Obs (site web), 17 déc. 2015. En ligne : https://www.nouvelobs.com/societe/etatd-urgence/20151217.OBS1596/marc-crepon-l-etat-d-urgence-est-contre-productif-a-longterme.html (consulté le 20 juillet 2016)

Debray, Régis (entretien avec Vincent Trémolet de Villers). 2016. « Une guerre civile? La "grosse blague”, aurait dit Flaubert », Le Figaro.fr, 3 oct. 2016). En ligne : http://www.lefigaro.fr/vox/ societe/2016/10/03/31003-20161003ARTFIG00274-regis-debray-une-guerre-civile-la-8220grosseblague8221-aurait-dit-flaubert.php (consulté le 17 septembre 2017)

Derrida, Jacques (entretien avec Jérôme-Alexandre Nielsberg). 2004. « Ni critique ni méthode, la déconstruction fait droit à des interprétations de lecture, d'écriture, qui sont autant d'événements. ", L'Humanité, 28 janv. 2004, p. 12 
Derrida, Jacques (entretien inédit avec Roger Pol-Droit). 2014. « Le philosophe est celui qui fait courir des risques », Le Point.fr, 9 novembre, 2014. http://www.lepoint.fr/livres/derrida-lephilosophe-est-celui-qui-fait-courir-des-risques-09-11-2014-1879805_37.php (consulté le 20 janvier 2017)

Derrida, Jacques : « Qu'est-ce que le terrorisme? (Entretien avec Giovanna Borradori). 2004. Le Monde diplomatique, février 2004, p. 16

Fleury, Cynthia (entretien Sophie Pujas). 2015. « Le terrorisme n'a pas le monopole du "surmoi" », Le Point presse magazine, 26 nov. 2015, n² 2255, p. 96-98

Gros, Frédéric. 2015. « Trop de sécuritaire tue la sécurité », Le Monde, 23 nov. 2015, p. 21

Habermas, Jürgen (entretien avec Nicolas Weill). 2015. « Le djihadisme, une forme moderne de réaction au déracinement ", Le Monde, 23 nov. 2015, p. 18

Le Goff, Jean-Pierre (entretien avec Alexandre Devecchio). 2015. «L'émotion du 11 janvier ne doit pas tout emporter », Le Figaro.fr, 23 déc. 2015. En ligne : http://www.lefigaro.fr/vox/societe/ 2015/12/23/31003-20151223ARTFIG00087-jean-pierre-le-goff-l-emotion-du-11-janvier-ne-doitpas-tout-emporter.php (consulté le 16 mars 2017)

Onfray, Michel (entretien avec Sébastien Le Fol). 2015. « La France doit cesser sa politique islamophobe ", Le Point.fr, 15 nov. 2015. En ligne : http://www.lepoint.fr/societe/michel-onfrayla-france-doit-cesser-sa-politique-islamophobe-15-11-2015-1981858_23.php (consulté le 16 mars 2017)

Onfray, Michel (entretien avec Virginie Larousse et Henri de Monvallier). 2016. «L'islam est devenu la religion de conversion d'une jeunesse désemparée ", Le Monde des religions, 24 fév. 2016, $n^{\circ} 76$, p. 68-71

Pol-Droit, Roger (entretien avec Denis Lafay). 2015. « Le vivre ensemble est malade mais il veut se soigner : voilà ce qu'enseigne le séisme Charlie Hebdo ", La Tribune Rhône-Alpes, $1^{\circ}$ fév. 2015, $n^{\circ}$ 124, pp. $4-13$

Pol-Droit, Roger. 2016. « La pensée du 11 septembre, quinze ans après », Les Echos, 9 sept. 2016, n 22273, p. 12

Pape François (entretien Jean-Marie Guénois). 2014. « L'appel du Pape aux musulmans contre le terrorisme islamique », Le Figaro.fr, 30 nov. 2014. En ligne : http://www.lefigaro.fr/actualitefrance/2014/11/30/01016-20141130ARTFIG00214-l-appel-du-pape-aux-musulmans-contre-leterrorisme-islamique.php (consulté le 18 juillet 2016)

\section{NOTES}

1. Ce corpus a été choisi pour des raisons que nous verrons plus loin. La liste des entretiens étudiés se trouve à la fin de cet article.

2. Il y a toute une littérature sur le journaliste comme "porte-parole ", on pourra notamment se référer à Michel Marccocia pour cette question.

3. C'est nous qui soulignons, dans cet extrait comme dans ceux qui suivent. 


\section{RÉSUMÉS}

La parole philosophique dans les médias a donné lieu à plusieurs recherches portant sur la figure de l'intellectuel ou du "penseur». Ces études adoptent des points de vue historiques ou sociologiques mais ne proposent pas une analyse, dans une perspective discursive, de la manière dont cette parole est concrètement mobilisée dans le texte journalistique lors des entretiens. Nous nous proposons ici d'analyser ce genre de discours en cherchant à dégager ses spécificités. Nous nous demandons en particulier quel type de question on adresse au philosophe lors d'un entretien de presse et si on s'entretient d'une manière particulière avec lui quand il est interpellé sur une question brûlante de l'actualité. Cette étude nous permettra de nous interroger sur la place du philosophe dans les médias en France. A cette fin, nous analysons divers textes récents où des philosophes parlent des attaques terroristes. Il s'agira enfin de montrer la spécificité les interventions des philosophes dans la presse par rapport à celles d'autres experts comme les sociologues et les politistes.

The philosophical speech in the media has already been studied through the lens of the intellectual or the "thinker". These studies adopt an historical or sociological point of view, but do not analyze from a discursive point of view the way this speech is concretely used in the journalistic texts or during interviews. In this paper, I propose to analyze the philosopher's speech in the media and to show its specificities. What types of questions is the philosopher asked during press interviews? Is the manner of interviewing specific when s/he is questioned about a burning news issue? This analysis reflects upon the philosopher's place in the contemporary French media. To this end, I examine various recent texts where philosophers speak about terrorist attacks, comparing the way they speak with that of other experts such as sociologists and political scientists.

\section{INDEX}

Keywords : discourse analysis, philosophical discourse, interviews print press

Mots-clés : analyse du Discours, discours philosophique, entretien, texte de presse

\section{AUTEUR}

\section{MALIKA TEMMAR}

Université d'Amiens/ Céditec (Paris Est) 